\title{
Elevation predicts the functional composition of alpine plant communities based on vegetative traits, but not based on floral traits
}

\author{
Robert R. Junker $^{1}$ (D) . Anne-Amélie C. Larue-Kontić ${ }^{1}$
}

Received: 18 August 2017 / Accepted: 19 December 2017 / Published online: 12 January 2018

(C) The Author(s) 2018. This article is an open access publication

\begin{abstract}
The functional diversity and composition of plant traits within communities are tightly linked to important ecosystem functions and processes. Whereas vegetative traits reflecting adaptations to environmental conditions are commonly assessed in community ecology, floral traits are often neglected despite their importance for the plants' life cycle. The consideration of floral traits covers important aspects such as sexual plant reproduction and pollinator diversity, which remain unobserved in studies focussing on vegetative traits only. To test whether vegetative and floral traits differ in their responses to elevation, we measured morphological and chemical traits of plant species occurring in pastures at seven elevations in the Austrian Alps. Variation in functional composition was examined using the concept of $n$-dimensional hypervolumes and vector analysis. Our data show that vegetative and floral traits vary differently with the elevational gradient. Whereas vegetative traits changed in a predictable manner with elevation, floral traits did not specifically respond to elevation. Overall variation in vegetative traits mainly resulted from phenotypical differences between plants in different elevations, whereas total variation in floral traits was a result from a high variation within communities. The assessment of functional changes in vegetative and floral traits along mountain slopes thus reveals different patterns in plant responses to elevation and may help to generate testable hypotheses on functional responses to current climate warming.
\end{abstract}

Keywords Dynamic range boxes $\cdot$ Flowers $\cdot$ Functional overlap $\cdot n$-dimensional hypervolume $\cdot$ Plant phenotype $\cdot$ Vector analysis

\section{Introduction}

Plant communities vary along elevational gradients both taxonomically and regarding the characteristics of their traits, which reflects responses to changing temperature regimes and other factors (Sundqvist et al. 2013). A very conspicuous effect of elevation are treelines, where trees as dominant components of the plant composition are replaced by shrubs and herbaceous plants (Körner 2003). More subtle changes in the functional composition along elevational gradients also confirm that the conditions at different elevations

Electronic supplementary material The online version of this article (https://doi.org/10.1007/s00035-017-0198-6) contains supplementary material, which is available to authorized users.

Robert R. Junker

robert.junker@sbg.ac.at

1 Department of Biosciences, University Salzburg, Hellbrunnerstraße 34, 5020 Salzburg, Austria favour the establishment of plants with different trait characteristics (Read et al. 2014; Sundqvist et al. 2013; Swenson et al. 2011). For instance, Kichenin et al. (2013) showed that leaf area concertedly decreased in most plant species with increasing elevation, which was also reflected in a decreasing community weighted mean in this trait. Usually, multiple traits are required to fully assess functional community composition (Kraft et al. 2015; Spasojevic and Suding 2012). Data on various traits allow to comprehensively track functional changes and transitions in communities as response to environmental changes or as cause for changes in ecosystem processes (Allan et al. 2015; Tilman et al. 1997). Thus, knowledge on the dispersion of several traits along elevational gradients can be valuable to understand and predict responses of plant communities to climate warming (Barros et al. 2016; Read et al. 2014; Sundqvist et al. 2013).

In plant community ecology, mostly vegetative traits are considered (e.g., Cornwell and Ackerly 2009; Kraft et al. 2015) that are well suited to assess the plants' capability to cope with abiotic resources and conditions such as light, 
water, and temperature or to infer plant growth strategy and competitive abilities (Ackerly and Cornwell 2007; Funk et al. 2016; Siefert et al. 2015; Stubbs and Wilson 2004). Less well studied are floral traits that are important for the plants' sexual reproduction, which requires animal pollen vectors in zoophilous plants and was suggested to be of particular importance for alpine plant species (Fabbro and Körner 2004). The few studies that considered floral traits in a community-ecological context (e.g., Benadi et al. 2014; Junker et al. 2013; Runquist et al. 2016; Wolowski et al. 2017) suggest a contribution of these traits to plant community composition (Benadi 2015; Pauw 2013; Sargent and Ackerly 2007). Indeed, recent studies show that floral traits, such as morphology, colour, and scent shape plant-pollinator interactions, affect the diversity of flower visiting animals in communities, and explain the presence or absence of competition between plant species for pollinators (Carvalheiro et al. 2014; Junker et al. 2013, 2015; Junker and Parachnowitsch 2015; Kuppler et al. 2016; Larue et al. 2016). Thus, pollination and floral traits are directly linked to plant reproduction and fitness, which are fundamental processes related to biomass gain and species diversity in communities. Both vegetative and floral traits may reflect the plants' adaptation to the conditions at a given elevation, which would result in predictable changes of trait characteristics along an elevational gradient. Alternatively, vegetative and floral traits may facilitate resource partitioning among plant species that compete either for light, water, or nutrients or for pollinators. In this scenario, traits dispersion would be large within a given community. The major goal of this study is to test whether vegetative and floral traits differ in their responses to elevation and in trait dispersion within and between communities.

Information of the dispersion of several traits is important to fully capture the complexity of community responses to environmental gradients. The framework of $n$-dimensional hypervolumes considers, unlike other multivariate approaches, each trait equally, does not reduce the number of dimensions, and thus represents a direct representation of functional community composition (Barros et al. 2016; Carmona et al. 2016; Junker et al. 2016; Kuppler et al. 2017; Lamanna et al. 2014). Accordingly, hypervolumes have been shown to be a valuable approach to track changes in community composition (Barros et al. 2016). Although the framework of $n$-dimensional hypervolumes has been proposed 60 years ago to describe niches (Hutchinson 1957), just recently, it became accessible through the development of statistical approaches that calculate the size and overlap of $n$-dimensional hypervolumes (for description of the different available approaches, see Blonder et al. 2014; Carmona et al. 2016; Junker et al. 2016; Swanson et al. 2015). The available approaches differ in the definition of the geometry of hypervolumes, in the number of dimensions that can be considered, and in the assumptions on the underlying distribution of the data. Here, we use dynamic range boxes (Junker et al. 2016), which is a non-parametric approach to quantify the size and overlap of hypervolumes and thus returns meaningful results independent on the data distribution. Furthermore, this approach can be used on any number of dimensions and allows comparing results based on data sets that differ in the number of dimensions. In addition, to the calculations implemented in the R package $d y n R B$ (Junker et al. 2016), we also introduce vector analysis to the framework of $n$-dimensional hypervolumes to track the length and the directions of functional changes of plant communities along an elevational gradient.

Using data on measurements of vegetative and floral traits of 70 plant species encountered at seven elevations in the Austrian Alps, we tested the following predictions:

1. Because of their different functions in plant ecology, the functional composition of plant communities changes differently along the elevational gradient depending on whether vegetative or floral traits are considered.

2. Plant communities encountered at different elevations differ in their trait characteristics, i.e., communities occupy unique $n$-dimensional hypervolumes.

3. Mean functional positions of communities within the $n$-dimensional hypervolume change in a predictable manner along the elevational gradient.

4. Variation in functional traits within communities (i.e., variation in functional traits between plant species encountered at a given elevation expressed as size of the $n$-dimensional hypervolume occupied by a community) is smaller than between communities at different elevations. Accordingly, overlaps between $n$-dimensional hypervolumes of communities at different elevations are expected to be relatively small.

\section{Methods}

\section{Study site}

We studied seven communities between 1146 and $2750 \mathrm{~m}$ a.s.l. located along the Großglockner High Alpine road in the Austrian Alps in May, June, and July 2014. Communities were selected based on accessibility and to evenly cover the elevational gradient. Plot size differed between communities and was chosen to represent local plant communities (Supporting Information Table S1). The number of plant species was not affected by plot size (Pearson's product moment correlation: $t_{6}=1.11, r^{2}=0.17$ ). All plots were dominantly covered by herbaceous vegetation. All flowering plant species encountered in these communities were phenotyped based on vegetative and floral traits. To test whether results 
are biased by time of year (phenology), we visited one plot (1281 $\mathrm{m}$ a.s.l.) one time at the beginning of our field season (mid-May, hereinafter referred as $1281 \mathrm{a}$ ), and one time at the end of our field season (mid-July, $1281 \mathrm{~b}$ ). Information on plot size and the number of flowering species is given in Supporting Information Table S1.

\section{Phenotyping of plants}

For all flowering plant species encountered in the plots, we quantitatively measured morphological (both vegetative and floral), and chemical traits (floral scents) as well as the number of flowering units as a surrogate for plant abundance. The traits considered have been chosen based on their importance for physiological processes and adaption to environmental conditions (Stubbs and Wilson 2004) or their important function in shaping plant-pollinator interactions (Junker et al. 2013; Junker and Parachnowitsch 2015; Larue et al. 2016). Per plant species and per plot, we recorded $n=7$ vegetative traits and $n=6$ floral traits. Plant individuals were sampled in the field and transported in a cooler to the lab where measurements started within $1 \mathrm{~h}$ after picking. For $n=5$ individuals per plant species and per plot, we measured the following vegetative traits to the nearest $1 \mathrm{~mm}$ using a calliper: leaf widths, leaf lengths, and thickness. Leaf area was obtained by scanning individual leaves. Numbers of pixels per leaf were compared to number of pixels of a $1 \mathrm{~cm}^{2}$ reference using the freeware fiji imageJ. The specific leaf weight was calculated by dividing the leaf weight [mg] by leaf area $\left[\mathrm{cm}^{2}\right]$ and the leaf lobation was calculated using the following formula: $(\pi \times 0.5 \times$ leaf length $\times 0.5 \times$ leaf width)/leaf area. Plant height was measured to the nearest $1 \mathrm{~mm}$ using a ruler directly in the field. For $n=7$ individuals per plant species and per plot, we also measured the following floral traits to the nearest $1 \mathrm{~mm}$ using a calliper: the display size of flowers, the width and depth of the nectar tubes, the position of anthers (defined as the distance between the flower surface and the anthers, negative values indicate that anthers are inside of the nectar tube), and the flower inclination, that is defined as the vertical inclination of flowers. Display size, the width, and depth of the nectar tubes as well as the position of anthers were measured to the nearest $1 \mathrm{~mm}$ using a calliper. The flower inclination was measured using a triangle ruler directly in the field. Morphological measurements were performed using a binocular microscope (Leica Microsystems, Vienna, Austria).

Floral volatiles were collected from all plant species per plot using dynamic headspace sampling methods directly in the field (Larue et al. 2016). Flowers were enclosed into scentless polyethylene tetraphthalate (PET) oven bags (Toppits ${ }^{\circledR}$ Cofresco Fischhalteprodukte GmbH \& Co. KG, Minden, Germany) for $45 \mathrm{~min}$, and afterward, the enriched headspace was sampled for $2 \mathrm{~min}$ into volatile traps with a flow rate of $200 \mathrm{ml} \mathrm{min}{ }^{-1}$. The scent traps were filled with a mixture of $1.5 \mathrm{mg}$ Tenax-TA (mesh 60-80; Supelco, Germany) and $1.5 \mathrm{mg}$ Carbotrap B (mesh 20-40; Supelco, Germany). Floral scent bouquets were analysed using an automatic thermal desorption system (TD, model TD-20, Shimadzu, Japan) coupled with gas chromatography/mass spectroscopy (GC-MS, model QP2010 Ultra EI, Shimadzu, Japan). The gas chromatograph was equipped with a ZB-5 column (Zebron ZB-5, 5\% phenyl polysiloxane, length $60 \mathrm{~m}$, inner diameter $0.25 \mathrm{~mm}$, film thickness $0.25 \mu \mathrm{m}$, Phenomenex, Newport Beach, USA). Carrier gas flow (helium) was set to $1.5 \mathrm{ml} \mathrm{min}{ }^{-1}$ and the oven temperature was hold for $1 \mathrm{~min}$ at $40{ }^{\circ} \mathrm{C}$ and rose then with $6{ }^{\circ} \mathrm{C} \mathrm{min}{ }^{-1}$ until the maximum of $250{ }^{\circ} \mathrm{C}$ was reached. The interface of the mass spectroscopy was $260{ }^{\circ} \mathrm{C}$ and ion trap worked at $200{ }^{\circ} \mathrm{C}$. Chromatograms and mass spectra were analysed with the GCMSsolutions software (Version 2.72, Shimadzu Corporation). All peaks, which occurred in the scent samples but not in control samples, were classified as authentic floral volatiles. Comparison of compounds in floral scent samples and control was based on Kovats' retention indices and mass spectra. Total peak area was divided by the number of flowers. Scent emission per plant species and hour was calculated.

\section{n-dimensional hypervolumes occupied by plant communities}

To characterise the functional composition of plant communities in plots $p$ as well as to quantify the changes in functional composition between plots, we adopted the concept of $n$-dimensional hypervolumes sensu Hutchinson (1957). In an $n$-dimensional hypervolume, each (vegetative or floral) trait is represented by one dimension and each plant species is characterized by one point within this coordinate system ( $n=$ total number of traits considered $T)$. Accordingly, the functional composition of a plant community found in plot $p$ is defined by the distribution of the plant species present in a community within the coordinate system. Thus, each plant community occupies a unique trait space in the $n$-dimensional hypervolume. The abundance weighted mean in the trait characteristics within a community defines the mean functional position of each community. First, we quantified the size $\operatorname{vol}\left(p_{n}\right)$ and overlap $\operatorname{port}\left(p_{n}, p_{m}\right)$ of the community-specific trait spaces using the approach dynamic range boxes (dynRB, Junker et al. 2016), which is a robust non-parametric approach to quantify the size and overlap of $n$-dimensional hypervolumes. dynRB accounts for the distribution of the data within their range, while no assumptions on the underlying distribution are required. Furthermore, using $d y n R B$, the abundance of plant species can be considered. We defined the trait space of communities as the 
$n$-dimensional hypervolume occupied by the plant species $j$ present in a plot $p$ weighted by the abundance of the plant species $j$. Abundance $a_{p j}$ was first transformed by $a_{p j}{ }^{\prime}=$ $\log \left(a_{p j}+1\right) \times 10$, and then, $a_{p j}{ }^{\prime}$ was rounded to the nearest integer $r\left(a_{p j}{ }^{\prime}\right)$, which is required by $d y n R B$ to consider plant abundance (compare to Kuppler et al. 2017). Each value of trait $t$ of plant species $j$ was $r\left(a_{p j}{ }^{\prime}\right)$ times added to the vector defining the functional position of the plant community in plot $p$. Thus, for each plot $p$ and trait $t$, we obtained a vector with the length $V=\sum_{j=1}^{J} r\left(a_{p j}^{\prime}\right)$ with $J$ being the total number of plant species in plot $p$. Thus, the $n$-dimensional hypervolume occupied by the plants species $j$ present in a plot $p$ (which is the functional composition of a plant community) was expressed as a matrix with $V$ rows and $T$ columns with $T$ as the total number of traits considered. Using this data frame, we calculated the size $\operatorname{vol}\left(p_{n}\right)$ of each plot $p_{n}$ and pairwise overlap $\operatorname{port}\left(p_{n}, p_{m}\right)$ of plots $p_{n}$ and $p_{m}$. Size $\operatorname{vol}\left(p_{n}\right)$ and overlaps $\operatorname{port}\left(p_{n}, p_{m}\right)$ were calculated using standard settings in function dynRB_ $V P a($ data) implemented in the $\mathrm{R}$ package dynRB (Junker et al. 2016). Results are displayed using either 'product' as aggregation method, or 'gmean' as aggregation method facilitating comparisons between vegetative and floral traits despite different numbers of traits considered. Dynamic range boxes $d y n R B$ calculates volumes (interval length in the one-dimensional setting) and overlaps for each coordinate (i.e., trait) of the $n$-dimensional hypervolume separately and subsequently aggregates these values over all dimensions $n$ (Junker et al. 2016). The aggregation method 'product' calculates the geometric volume delineated by the interval lengths of each dimension and thus reflects Hutchinson's (1957) $n$-dimensional hypervolme. However, by multiplying the coordinate-wise values, the hypervolume becomes smaller with increasing number of dimensions, which prevents a direct comparison of volumes and overlaps of data sets consisting of different numbers of dimensions. Therefore, dynRB also provides the aggregation method 'gmean', which considers the geometric mean of the coordinate-wise values and is thus independent on the number of dimensions. In our study, we considered $n=7$ vegetative traits and $n=6$ floral traits and, therefore, decided to present some of the results using the aggregation method 'gmean' to facilitate the comparability of these trait groups. As co-variation of traits may affect the results of dynamic range boxes, we tested for correlations between traits (Supporting Information Figs. $\mathrm{S} 1, \mathrm{~S} 2)$. The size of $n$-dimensional hypervolumes defined by $\operatorname{vol}\left(p_{n}\right)$ represents the proportion of the total trait space occupied by all species in all elevations covered by the volume of one community. Therefore, $\operatorname{vol}\left(p_{n}\right)$ is a relative measure and is thus comparable between hypervolumes based on different groups of traits (e.g., vegetative and floral traits). The same is true for the overlap of hypervolumes $\operatorname{port}\left(p_{n}, p_{m}\right)$.

Overlaps $\operatorname{port}\left(p_{n}, p_{m}\right)$ of $n$-dimensional hypervolumes do inform about the similarity of the functional composition of plant communities but do not reveal the magnitude and direction of shifts of community weighted mean positions within $n$-dimensional hypervolumes. To quantify these features, we applied vector analysis to calculate the length $l\left(\mathbf{u}_{\mathbf{p}}\right)$ [dimensionless] of the vectors $\mathbf{u}_{\mathbf{p}}$ describing the magnitude of the shifts between adjacent elevations. Each vector $\mathbf{u}_{\mathbf{p}}$ has the community weighted mean (cwm) position of the plant species in one plot $p$ as starting point and the cwm position of the plant species in the next higher elevation $p+1$ as end point. In addition, we calculated the deviation of the unit directions of vectors $\mathbf{u}_{\mathbf{1}}, \mathbf{u}_{\mathbf{p}}, \ldots, \mathbf{u}_{\mathbf{P}}(P$ being the number of plots observed -1$)$ in terms of the angle $\varphi_{u, u+1}$ $\left[{ }^{\circ}\right.$ ] between unit directions $\mathbf{u}_{\mathbf{p}}$ and $\mathbf{u}_{\mathbf{p}+1} \cdot \varphi_{u, u+1}=0^{\circ}$ indicates that vectors $\mathbf{u}_{\mathbf{p}}$ and $\mathbf{u}_{\mathbf{p}+\mathbf{1}}$ point towards the same direction and $180^{\circ}$ that these vectors point towards opposite direction. Narrow angles thus indicate that mean functional composition of communities shifts in a predictable manner along the elevation.

Data analyses were performed using R: A language and environment for statistical computing (R Core Team 2016).

\section{Results}

In total, we measured the traits of $n=70$ plant species in seven plots based on seven vegetative and six floral traits. On average, $n$-dimensional hypervolumes defined by vegetative traits, which is the hypervolume with $n=7$ vegetative traits as dimensions occupied by the plant species present in each community, were smaller than those defined by $n=6$ floral traits (Fig. 1a, paired $t$ test: $t_{7}=3.24, p=0.014$, based on $\operatorname{vol}\left(p_{n}\right)$ calculated using 'gmean' as aggregation method to enable comparison despite different numbers of traits considered). Likewise, overlap port $\left(p_{n}, p_{m}\right)$ between communities were smaller when calculated for vegetative traits than for floral traits (Fig. 1b, paired $t$ test: $t_{55}=6.27, p<0.001$, based on 'gmean' as aggregation method). The functional composition of vegetative traits in plant communities at different elevations showed little functional overlap $\operatorname{port}\left(p_{n}, p_{m}\right)$ in adjacent elevations. In contrast, Communities in distant elevations often did not functionally overlap (Fig. 2a). This finding is also supported by the strong and significant negative correlation between the difference in elevation between communities $p_{n}$ and $p_{m}$ and their functional overlap based on vegetative traits (Fig. 1c, Pearson's product-moment correlation: $\left.t_{54}=-6.38, p<0.001, r^{2}=0.43\right)$. As an exception, the community at the lowest elevation (1146 $\mathrm{m}$ a.s.l.) overlapped stronger with communities at middle elevations (1742 and $2086 \mathrm{~m}$ a.s.1.) than with communities in adjacent elevations 


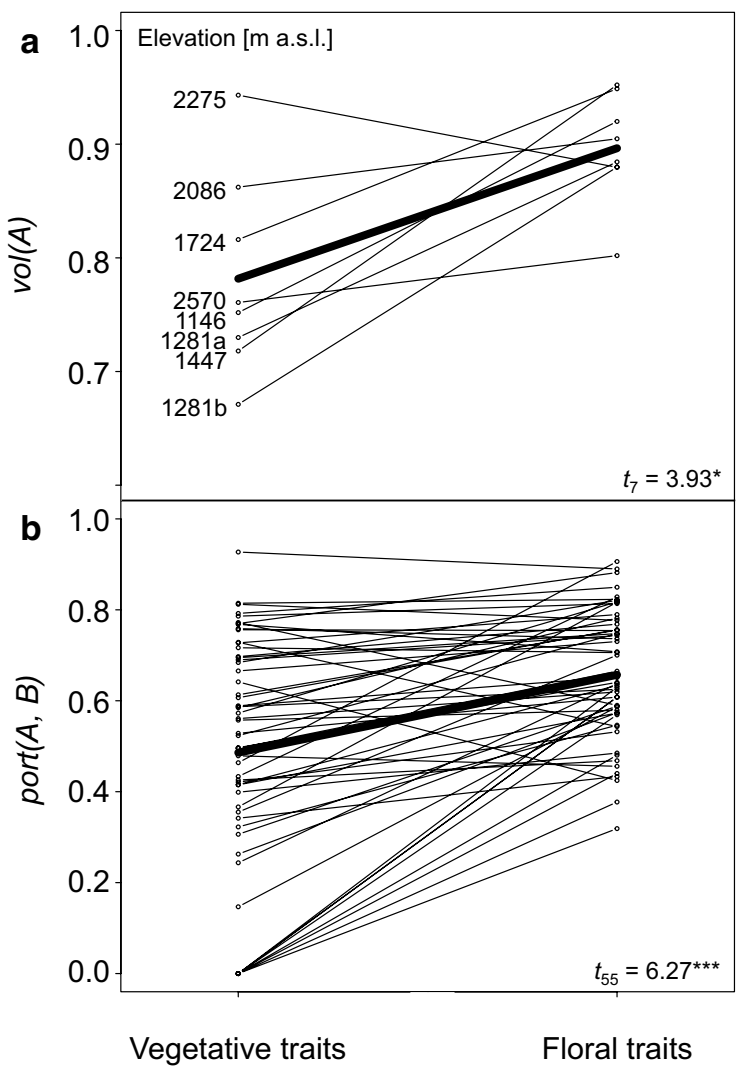

Fig. 1 Size (a) and overlap (b, c) of $n$-dimensional hypervolumes occupied by communities at different elevations. Functional size $\operatorname{vol}\left(p_{n}\right)$ and overlap $\operatorname{port}\left(p_{n}, p_{m}\right)$ were calculated using dynamic range boxes (Junker et al. 2016) using standard parameters and the aggregation method 'gmean' allowing for direct comparison of $n$-dimensional hypervolumes based on $n=7$ vegetative and $n=6$ floral traits. a Thin lines link sizes $\operatorname{vol}\left(p_{n}\right)$ of hypervolumes based on vegetative traits with sizes based on floral traits at the same elevation. The bold line links the mean size based on vegetative traits with the mean size based on floral traits. On average, hypervolumes based on floral traits are larger than those based on vegetative traits (paired $t$ test). b Thin lines link overlaps $\operatorname{port}\left(p_{n}, p_{m}\right)$ based on vegetative traits with over-

(1281 and 1447 m a.s.l., Fig. 2a). Whereas plant communities of distant elevations did functionally not overlap based on vegetative traits [i.e., $\operatorname{port}\left(p_{n}, p_{m}\right)=0$ ], functional overlap based on $n=6$ floral traits occurred between all communities $\left[\operatorname{port}\left(p_{n}, p_{m}\right) \geq 0.033\right.$, Fig. 2b)]. Likewise, elevational distance did only weakly affect the functional overlap based on floral traits (Fig. 2b), indicated by the significant but weak correlation between the difference in elevation between communities $p_{n}$ and $p_{m}$ and their functional overlap based on floral traits (Fig. 1c, Pearson's product-moment correlation: $\left.t_{54}=-2.22, p=0.031, r^{2}=0.08\right)$. Note that overlaps $\operatorname{port}\left(p_{n}\right.$, $p_{m}$ ) (aggregated by 'product') based on vegetative and floral traits cannot directly be compared due to the different numbers of dimensions, which affect the absolute values of $\operatorname{port}\left(p_{n}, p_{m}\right)$. For a direct comparison, see Fig. S4 showing

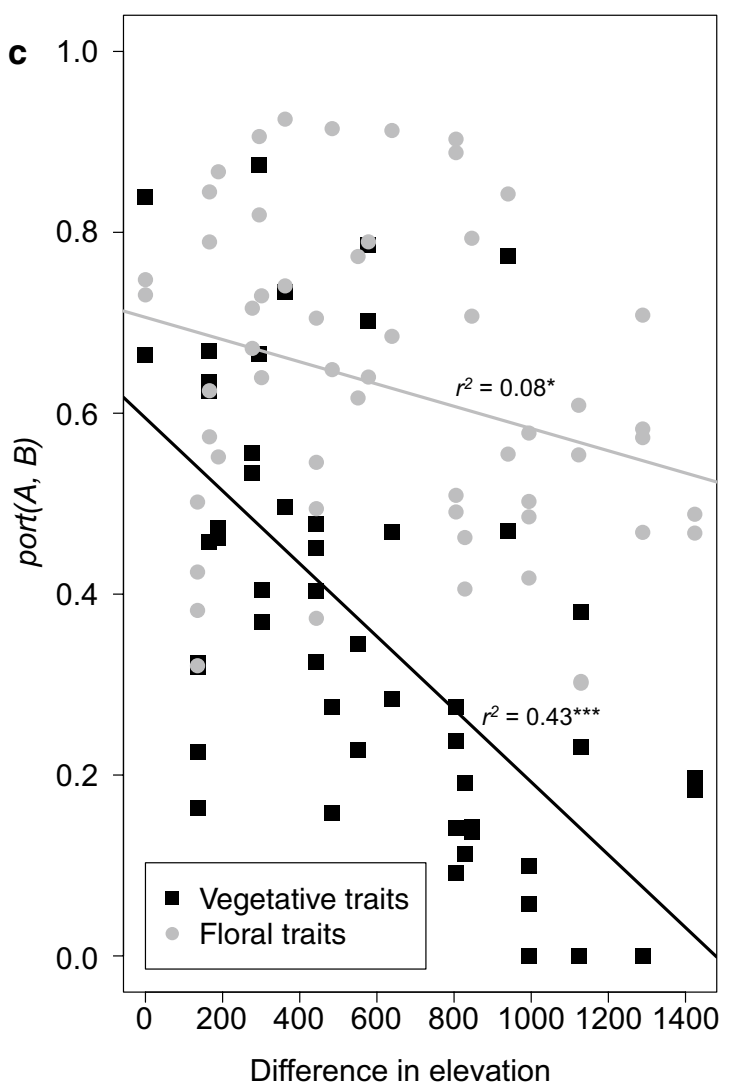

laps based on floral traits of the same pair of communities $p_{n}$ and $p_{m}$ at different elevations. The bold line links the mean overlap based on vegetative traits with the mean overlap based on floral traits. On average, overlaps based on floral traits are larger than those based on vegetative traits (paired $t$ test). Note that $\operatorname{port}\left(p_{n}, p_{m}\right)$, which is $p_{n}$ in $p_{m}$ or the portion of the trait space of $p_{m}$ that is covered by $p_{n}$, is not the same as $\operatorname{port}\left(p_{m}, p_{n}\right)$, because overlaps are, by construction, asymmetric. c Overlaps $\operatorname{port}\left(p_{n}, p_{m}\right)$ based on vegetative traits (black squares) or floral traits (grey circles) as a function of the difference in the elevation between communities $p_{n}$ and $p_{m}$. Overlaps $\operatorname{port}\left(p_{n}, p_{m}\right)$ based on aggregation method 'gmean' were used for the dependent variable

results based on aggregation method 'gmean' (geometric mean), which corrects for different numbers of dimensions (patterns remain the same; Supporting Information Fig. S4).

The visualizations of three-dimensional hypervolumes based on vegetative (Fig. 3a) and floral traits (Fig. 3b) support the findings on the differences in volume of and overlap between communities between the trait groups. Note that we selected those three traits for visualization that separate the communities most strongly, i.e., those with the lowest overlap $\operatorname{port}\left(p_{n}, p_{m}\right)$ between communities. Visual inspection of three-dimensional hypervolumes supports the finding (see Fig. 2) that the overall vegetative trait space occupied by all plant species of the regional species pool is the result of functional differences between communities. This finding is well reflected by the relative small sizes $\operatorname{vol}\left(p_{n}\right)$ of and 


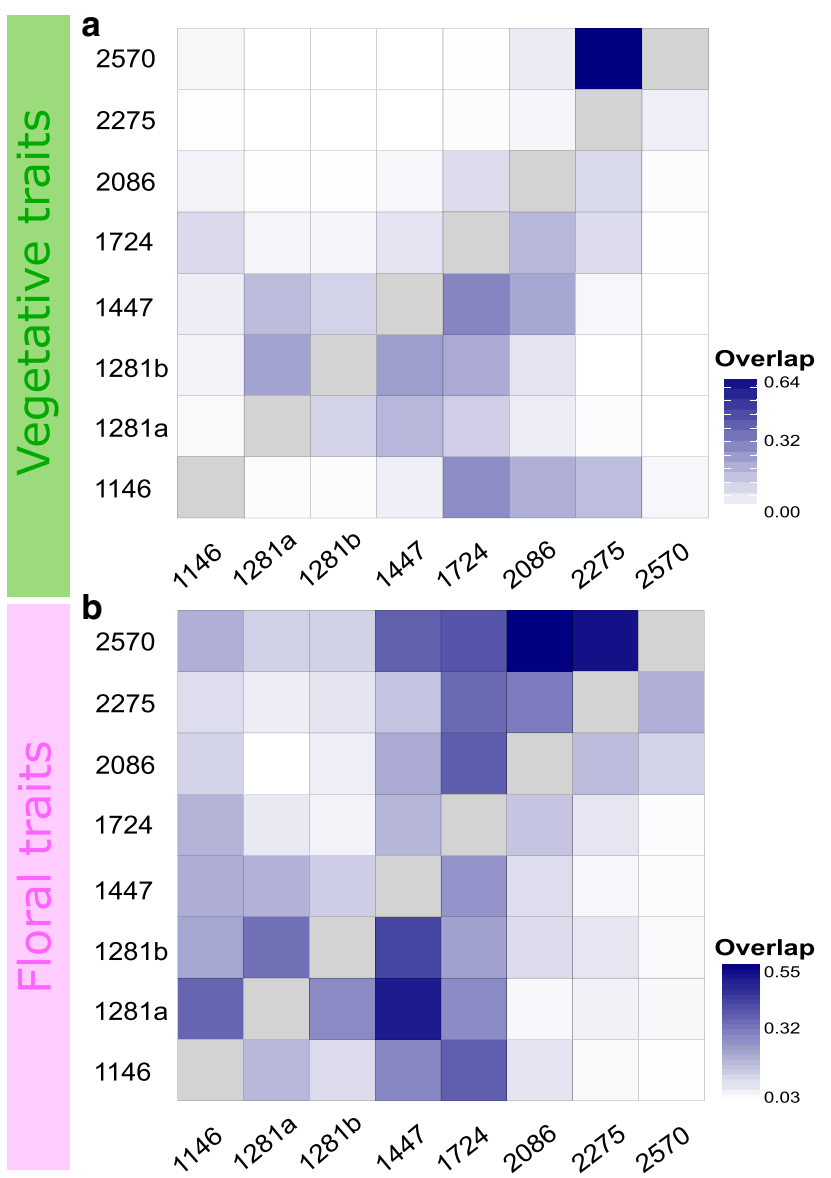

Fig. 2 Functional overlap of plant communities at different elevations. Heatmaps depict the overlaps $\operatorname{port}\left(p_{n}, p_{m}\right)$ of plots $p_{n}$ and $p_{m}$ based on a $n=7$ vegetative or $\mathbf{b} n=6$ floral traits at different elevations (m a.s.l). Functional overlap port $\left(p_{n}, p_{m}\right)$ was calculated using dynamic range boxes (Junker et al. 2016) using standard parameters and the aggregation method 'product' (results using the geometric mean 'gmean' as aggregation method are shown in Supporting Information Fig. S4, facilitating a direct comparison of values). Light colours indicate little or no overlap; dark colours indicate strong overlap (note different scales for each heatmap). Note that $\operatorname{port}\left(p_{n}, p_{m}\right)$, which is $p_{n}$ in $p_{m}$ or the portion of the trait space of $p_{m}$ that is covered by $p_{n}$, is not the same as $\operatorname{port}\left(p_{m}, p_{n}\right)$, because overlaps are, by construction, asymmetric. The heatmap is visualizing the proportion of trait space of communities denoted in the $x$-axis that is overlapped by the trait space of the communities denoted in the $y$-axis

overlaps $\operatorname{port}\left(p_{n}, p_{m}\right)$ between communities (Figs. 1, 2). In contrast, the overall expansion of the floral trait space largely results from interspecific differences within communities, reflected by larger sizes $\operatorname{vol}\left(p_{n}\right)$ of and overlaps $\operatorname{port}\left(p_{n}, p_{m}\right)$ between communities (Figs. 1, 2).

The length in shifts between adjacent elevations in terms of vector length $l\left(\mathbf{u}_{\mathbf{p}}\right)$ of vector $\mathbf{u}_{\mathbf{p}}$, which is the vector with community weighted mean (cwm) position of the plant species in one plot as starting point and the cwm position of the plant species in the next higher elevation as end point, was

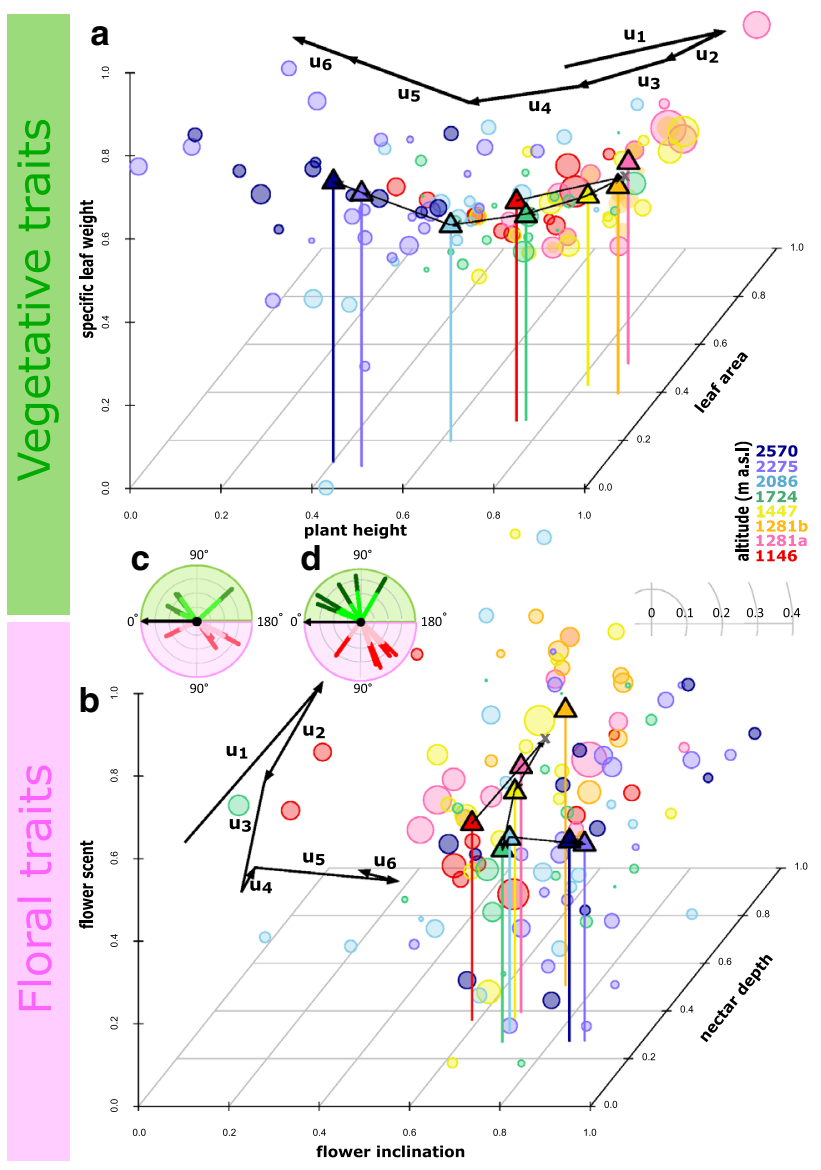

Fig. 3 Three-dimensional hypervolumes occupied by plant communities at different elevations with either a vegetative traits or $\mathbf{b}$ floral traits as dimensions. Each circle represents a plant species present in one of the eight communities. Elevation of communities is colour-coded. Size of circles is proportional to local abundance of plant species. Communityweighted mean (cwm) position of communities is shown as triangle. The plant species in the community at $1281 \mathrm{~m}$ a.s.l. have been phenotyped two times (May and July) to test whether phenology affects the functional diversity and composition of plant communities. Because we wanted to track the shifts in functional composition based on elevation independent of phenology (and because cwms of both communities are positioned in relatively close proximity within the hypervolume), we used the mean position of the two cwms (indicated by ' $\mathrm{x}$ ') for the following analysis. Vectors $\mathbf{u}_{\mathbf{1}}, \mathbf{u}_{\mathbf{p}}, \ldots, \mathbf{u}_{\mathbf{P}}$ with cwm position of community $p$ as start point and cwm position of community $p+1$ as end point show length and direction of functional shift. The signatures of the shifts are also shown as enlargement. c, $\mathbf{d}$ Length $l\left(\mathbf{u}_{\mathbf{p}}\right)$ of vectors $\mathbf{u}_{\mathbf{1}}, \mathbf{u}_{\mathbf{p}}, \ldots, \mathbf{u}_{\mathbf{p}}$ and deviation of the unit directions of vectors $\mathbf{u}_{1}, \mathbf{u}_{\mathbf{p}}, \ldots, \mathbf{u}_{\mathbf{P}}$ in terms of the angle $\varphi_{u, u+1}$ in the $n$-dimensional hypervolume are shown in radial plots. Scale for length $l$ of vectors is shown on the right. Lines in $\mathbf{c}$ depict length $l\left(\mathbf{u}_{\mathbf{p}}\right)$ and deviation of the unit directions $\varphi_{u, u+1}$ in the three-dimensional hypervolume, as shown in $\mathbf{a}$ (green) and $\mathbf{b}$ (pink). Dark part of vector is length $l$ of $\mathbf{u}_{\mathbf{p}}$, light part is $l$ of $\mathbf{u}_{\mathbf{p}+\mathbf{1}}$. Lines in d depict $\varphi_{u, u+1}$ of the $n$-dimensional hypervolume with all vegetative (green) and floral (pink) traits measured as dimensions. The lines in $\mathbf{c}$ are (ordered by increasing $\varphi_{u, u+1}$ ): vegetative: $\varphi_{u 3, u 4}$ $<\varphi_{u 4, u 5}<\varphi_{u 2, u 3}<\varphi_{u 5, u 6}<\varphi_{u 1, u 2}$. Floral: $\varphi_{u 2, u 3}<\varphi_{u 4, u 5}<\varphi_{u 3, u 4}<\varphi_{u 1, u 2}<$ $\varphi_{u 5, u 6}$. The lines in $\mathbf{d}$ are (ordered by increasing $\varphi_{u, u+1}$ ): vegetative: $\varphi_{u 3, u 4}$ $<\varphi_{u 4, u 5}<\varphi_{u 2, u 3}<\varphi_{u 5, u 6}<\varphi_{u 1, u 2}$. Floral: $\varphi_{u 2, u 3}<\varphi_{u 3, u 4}<\varphi_{u 4, u 5}<\varphi_{u 5, u 6}<$ $\varphi_{u 1, u 2}$. Animated three-dimensional hypervolumes facilitating a better evaluation of the spatial configuration of the plant species and cwms are given in Supporting Information Fig. S5a (vegetative traits) and Fig. S5b (floral traits) 
longer in the $n$-dimensional hypervolume based on vegetative traits than in the $n$-dimensional hypervolume based on floral traits (paired $t$ test: $t_{5}=2.94, p=0.032$, Fig. 3c, d). In addition, the functional changes based on vegetative traits in community-weighted mean positions of plant species along the elevational gradient occured in a predictable manner (Fig. 3). In contrast, functional changes in floral traits appear to be random and not related to the elevational gradient (Fig. 3). Vector analysis supports this finding as the deviation of the unit directions of vectors $\mathbf{u}_{1}, \mathbf{u}_{\mathbf{p}}, \ldots, \mathbf{u}_{\mathbf{P}}$ in terms of the angle $\varphi_{u, u+1}$ in the $n$-dimensional hypervolume with vegetative traits as dimensions are mostly clearly below $90^{\circ}$. This means that the unit direction of the vector $\mathbf{u}_{\mathbf{p}+\mathbf{1}}$ points towards a similar direction as the vector $\mathbf{u}_{\mathbf{p}}$, which are two consecutive vectors with community-weighted mean (cwm) position of the plant species in one plot as starting point and the cwm position of the plant species in the next higher elevation as end point (Fig. 3a, c, d). In contrast, angles $\varphi_{u, u+1}$ denote the deviations of consecutive vectors $\mathbf{u}_{\mathbf{p}}$ and $\mathbf{u}_{\mathbf{p}+1}$ in the $n$-dimensional hypervolume with floral traits as dimensions mostly were above $90^{\circ}$ (Fig. 3b-d). This means that shifts in functional composition of floral traits are not predictable by an elevational gradient. Accordingly, angles $\varphi_{u, u+1}$ based on vegetative traits tended to be smaller than the same angles $\varphi_{u, u+1}$ based on floral traits (paired $t$ test: $t_{4}=2.53, p=0.065$, Fig. 3). Note that Fig. 3c, d shows the length and deviation of unit directions of two consecutive vectors one time for the three-dimensional hypervolumes, as shown in Fig. 3a, b (Fig. 3c) and one time for the sevendimensional hypervolume with vegetative traits as dimensions and the six-dimensional hypervolume with floral traits as dimensions (Fig. 3d). Vector analysis in $n$-dimensional hypervolumes with all vegetative and floral traits as dimensions support the findings found in three-dimensional hypervolumes (Fig. 3c, d). Accordingly, vector lengths $l\left(\mathbf{u}_{\mathbf{p}}\right)$ and angles $\varphi_{u, u+1}$ based on either three vegetative or floral traits strongly correlated with the same vector lengths $l\left(\mathbf{u}_{\mathbf{p}}\right)$ and angles $\varphi_{u, u+1}$ based on $n$ traits (Pearson's product-moment correlation: $l\left(\mathbf{u}_{\mathbf{p}}\right): t_{4}=3.1, p=0.036, r^{2}=0.71 ; \varphi_{u, u+1}$ : $t_{3}=4.08, p=0.027, r^{2}=0.85$ ). Thus, the three-dimensional hypervolume, as shown in Fig. 3, is a good representation of the $n$-dimensional hypervolume. The patterns observed in the $n$-dimensional hypervolume are supported by individual traits: Most vegetative traits changed with the elevational gradient, i.e., on average, plants became smaller, the size of leaves decreased, and leaves became thicker and increased in specific leaf weight with increasing elevation (Supporting Information Fig. S3a-h, Tables S2, S3). Most floral traits (total flower scent emission, pollen position, and the depth of nectar tubes) varied independent on elevation except for flower inclination, which increased with increasing altitude, and the width of the nectar tubes, which decreased with increasing altitudes (Supporting Information Fig. S3i-n, Tables S2, S3).

\section{Discussion}

The functional composition of vegetative and floral traits varied in different ways with the elevational gradient in the Austrian Alps. When considering vegetative traits, plant communities responded as predicted to the elevational gradient: Each community occupied a unique $n$-dimensional hypervolume; the mean functional composition of trait characteristics changed in a predictable and concerted manner; and due to the relative small size of $n$-dimensional hypervolumes and their unique position, overlap between the $n$-dimensional hypervolumes of communities at different elevations was relatively small. The lowest plant community at $1146 \mathrm{~m}$ a.s.l., however, deviated from the general trend observed in the other communities as its functional composition was more similar to plant communities in higher elevations (most of all the plant community at $1724 \mathrm{~m}$ a.s.l.). This deviation from the general trend may be attributed to differences in land-use intensity (repeated mowing per year), resulting in, e.g., lower plant height and smaller leaf area compared to plant communities that had not been mowed or grazed until our recordings. In contrast to results based on vegetative traits, $n$-dimensional hypervolumes occupied by communities based on floral traits were larger and, therefore, the overlap between the hypervolumes was larger, too. Furthermore, we did not detect predictable changes in community composition but rather random directions of changes in mean position of communities. Therefore, our data confirm our first prediction that vegetative and floral traits differ in their responses to elevational gradients.

The different responses of vegetative and floral traits to the elevational gradient may reflect the divergent ecological roles of both groups of traits. Characteristics of vegetative traits inform about the plants' adaptation to local climatic conditions, nutrient and water availability, life strategies, but also about adaptations to biotic factors such as herbivores (Cornelissen et al. 2003). We found that vegetative traits show a low within but a large between community variation. Accordingly, as stress level, availability of light, water, and heat, and other factors vary along elevational gradients (Körner 2003), it is not surprising that the plant communities change in their functional composition along the elevation gradient in a predictable and concerted manner, too. It has been shown that traits of different plant organs respond heterogeneously to environmental changes (Radville et al. 2016; Runquist et al. 2016). Following this finding, the large within and relatively low between community variation in floral traits 
suggest that these traits respond to different factors than vegetative traits. Accordingly, the flowers' morphology, colour, and scent emission are well known to mediate interactions with pollinators (Junker et al. 2013; Kuppler et al. 2016; Larue et al. 2016), as they either facilitate interactions by means of attractive traits and the provisioning of resources or decrease or prevent interactions by means of morphological barriers, inconspicuous appearances, or repellent scents (Junker 2016; Junker and Parachnowitsch 2015; Katzenberger et al. 2013; Papiorek et al. 2015; Stang et al. 2007). According to the importance of functional flower traits in determining the visitor spectrum of a plant species, it has been shown that a high functional diversity in floral traits supports high species richness in flower visitors (Junker et al. 2015; Potts et al. 2004). The consideration of both vegetative and floral traits thus provides a more detailed view on plant responses to environmental gradients. Ackerly and Cornwell (2007) conceptualized different patters of trait dispersion as alpha traits that segregate locally co-occurring species and as beta traits that inform about the species' position along a gradient. Our data suggest that variation in vegetative traits mostly resulted from differences between elevations, whereas overall variation in floral traits mostly resulted from differences within communities. Thus, sensu Ackerly and Cornwell (2007) vegetative traits can-on averagebe regarded as beta traits that inform about the species' position along a gradient. Floral traits, in contrast, can be regarded as alpha traits that differentiate co-occurring species. Thus, our study strongly reemphasizes the importance of a careful selection of traits in community-ecological studies aiming at inferring ecosystem properties and functions (Cadotte et al. 2013; Kraft et al. 2015; Spasojevic and Suding 2012).

Climate change along with other anthropogenic alterations of ecosystems has severe effects on organisms and communities and, therefore, on biodiversity and ecosystem processes (Bellard et al. 2012; Sala et al. 2000). To describe or predict such effects on a large-scale, elevational gradients represent long-term and large-scale study systems that help to extract such information (Sundqvist et al. 2013) and have been used as surrogate for climatic shifts ('space for time' approach, Benadi et al. 2014). However, note that plots along an elevational gradient differ in many factors, not only annual mean temperatures, such as edaphic conditions, precipitation, and radiation that have not been examined in our study. Therefore, 'space for time' approaches such as our study do not directly translate into community responses to climate warming, but provide valuable starting points for future experimental studies that specifically test the hypotheses generated in field observations. Our results showed that the overall composition of floral traits may only respond weakly, which may buffer negative effects of climate warming. However, individual traits such as flower inclination and nectar tube width correlated to elevation, which clearly changes resource accessibility for many flower visitors. Regardless the consequences for ecosystem properties and function, our data suggest that plant communities will change their functional composition as a result of climate warming.

Our study demonstrated that the functional composition of plant communities responds to an elevation gradient, suggesting that similar shifts may be expected as a consequence of climate warming. However, predictable and concerted shifts were only observed in traits that respond to environmental factors such as the availability of light, water, and heat (Körner 2003; Stubbs and Wilson 2004), i.e., the vegetative traits measured in our study. The overall composition of traits that mediate interactions with pollinators (i.e., floral traits) was largely unaffected by elevation, or at least did not change in a predictable manner. The consideration of both vegetative and floral traits in community ecology thus informs about different aspects of plant ecology. Inventories of species assemblages along elevational gradients together with comprehensive assessment of their phenotype are thus valuable tools to detect community-ecological patterns and to generate testable hypotheses on functional responses to current climate warming.

Acknowledgements Open access funding provided by Paris Lodron University of Salzburg. We thank Lisa Wiesmann for contributing to the field work of this study, Wolfgang Trutschnig for the help with statistical analysis, and Guaraci Duran Cordeiro, Stefan Dötterl, Inge Illich, Olivier Marcel Narcisse Larue, Irmgard Schäffler, Marcel Vorage, Kjirsten Wayman, the 'Haus der Natur', and the National Park Hohe Tauern for help in the field, for logistic support or for providing infrastructure. Jonas Kuppler and Martin Lechleitner provided valuable comments on a previous version of the manuscript. This work was funded by the Glockner-Öko-Fond and the Deutsche Forschungsgemeinschaft (DFG, JU2856/1-1).

Declaration of authorship RRJ conceived the study, RRJ and AACLK designed the study, AACLK and RRJ performed fieldwork, and RRJ and AACLK performed statistical analysis and wrote the manuscript.

\section{Compliance with ethical standards}

Conflict of interest The authors declare no conflict of interest.

Ethical statement The study is in compliance with ethical standards.

Data accessibility statement Data supporting the results are given in supporting information 2 .

Open Access This article is distributed under the terms of the Creative Commons Attribution 4.0 International License (http://creativecommons.org/licenses/by/4.0/), which permits unrestricted use, distribution, and reproduction in any medium, provided you give appropriate credit to the original author(s) and the source, provide a link to the Creative Commons license, and indicate if changes were made. 


\section{References}

Ackerly DD, Cornwell WK (2007) A trait-based approach to community assembly: partitioning of species trait values into within- and among-community components. Ecol Lett 10:135-145. https:// doi.org/10.1111/j.1461-0248.2006.01006.x

Allan E et al (2015) Land use intensification alters ecosystem multifunctionality via loss of biodiversity and changes to functional composition. Ecol Lett 18:834-843. https://doi.org/10.1111/ ele. 12469

Barros C, Thuiller W, Georges D, Boulangeat I, Münkemüller T (2016) $\mathrm{N}$-dimensional hypervolumes to study stability of complex ecosystems. Ecol Lett 19:729-742. https://doi.org/10.1111/ele.12617

Bellard C, Bertelsmeier C, Leadley P, Thuiller W, Courchamp F (2012) Impacts of climate change on the future of biodiversity. Ecol Lett 15:365-377. https://doi.org/10.1111/J.1461-0248.2011.01736.X

Benadi G (2015) Requirements for plant coexistence through pollination niche partitioning. P R Soc B 282:Artn 20150117. https://doi. org/10.1098/Rspb.2015.0117

Benadi G, Hovestadt T, Poethke HJ, Bluthgen N (2014) Specialization and phenological synchrony of plant-pollinator interactions along an altitudinal gradient. J Anim Ecol 83:639-650. https:// doi.org/10.1111/1365-2656.12158

Blonder B, Lamanna C, Violle C, Enquist BJ (2014) The n-dimensional hypervolume. Global Ecol Biogeogr 23:595-609 doi:Doi. https:// doi.org/10.1111/Geb.12146

Cadotte M, Albert CH, Walker SC (2013) The ecology of differences: assessing community assembly with trait and evolutionary distances. Ecol Lett 16:1234-1244. https://doi.org/10.1111/ Ele. 12161

Carmona CP, de Bello F, Mason NWH, Leps J (2016) Traits without borders: Integrating functional diversity across scales. Trends Ecol Evol 31:382-394. https://doi.org/10.1016/j.tree.2016.02.003

Carvalheiro L et al (2014) The potential for indirect effects between co-flowering plants via shared pollinators depends on resource abundance, accessibility and relatedness. Ecol Lett 17:1389-1399

Cornelissen JHC et al (2003) A handbook of protocols for standardised and easy measurement of plant functional traits worldwide. Aust J Bot 51:335-380. https://doi.org/10.1071/BT02124

Cornwell WK, Ackerly DD (2009) Community assembly and shifts in plant trait distributions across an environmental gradient in coastal. California Ecol Monogr 79:109-126. https://doi. org/10.1890/07-1134.1

Fabbro T, Körner C (2004) Altitudinal differences in flower traits and reproductive allocation. Flora 199:70-81. https://doi. org/10.1078/0367-2530-00128

Funk JL et al. (2016) Revisiting the Holy Grail: using plant functional traits to understand ecological processes. Biol Rev in press

Hutchinson GE (1957) Concluding remarks. Cold Spring Harbour Symp Quant Biol 22:415-427

Junker RR (2016) Multifunctional and diverse floral scents mediate biotic interactions embedded in communities. In: Blande JD, Glinwood RT (eds) Deciphering chemical language of plant communication, vol in press. Springer, Heidelberg

Junker RR, Parachnowitsch AL (2015) Working towards a holistic view on flower traits-how floral scents mediate plant-animal interactions in concert with other floral characters. J Indian Inst Sci 95:44-67 (0970-4140 Coden-JIISAD)

Junker RR, Blüthgen N, Brehm T, Binkenstein J, Paulus J, Schaefer HM, Stang M (2013) Specialization on traits as basis for the niche-breadth of flower visitors and as structuring mechanism of ecological networks. Funct Ecol 27:329-341

Junker RR, Blüthgen N, Keller A (2015) Functional and phylogenetic diversity of plant communities differently affect the structure of flower-visitor interactions and reveal convergences in floral traits. Evol Ecol 29:437-450. https://doi.org/10.1007/ s10682-014-9747-2

Junker RR, Kuppler J, Bathke A, Schreyer ML, Trutschnig W (2016) Dynamic range boxes - a robust non-parametric approach to quantify the size and overlap of niches and trait-spaces in $n$-dimensional hypervolumes. Methods Ecol Evol 7:1503-1513

Katzenberger TD, Lunau K, Junker RR (2013) Salience of multimodal flower cues manipulates initial responses and facilitates learning performance of bumblebees. Behav Ecol Sociobiol 67:1587-1599

Kichenin E, Wardle DA, Peltzer DA, Morse CW, Freschet GT (2013) Contrasting effects of plant inter- and intraspecific variation on community-level trait measures along an environmental gradient. Funct Ecol 27:1254-1261. https://doi. org/10.1111/1365-2435.12116

Körner C (2003) Alpine plant life: functional plant ecology of high mountain ecosystems, 2 edn. Springer, Berlin

Kraft NJB, Godoy O, Levine JM (2015) Plant functional traits and the multidimensional nature of species coexistence. Proc Natl Acad Sci USA 112:797-802. https://doi.org/10.1073/Pnas.1413650112

Kuppler J, Höfers M, Wiesmann L, Junker RR (2016) Time-invariant differences between plant individuals in interactions with arthropods correlate with intraspecific variation in plant phenology, morphology and floral scent. New Phytol 210:1357-1368. https:// doi.org/10.1111/nph.13858

Kuppler J, MK H, Trutschnig W, Bathke A, Eiben J, Daehler C, Junker R (2017) Exotic flower visitors exploit large floral trait spaces resulting in asymmetric resource partitioning with native visitors. Funct Ecol (in press)

Lamanna $\mathrm{C}$ et al (2014) Functional trait space and the latitudinal diversity gradient. Proc Natl Acad Sci USA 111:13745-13750. https:// doi.org/10.1073/pnas.1317722111

Larue AAC, Raguso RA, Junker RR (2016) Experimental manipulation of floral scent bouquets restructures flower-visitor networks in the field. J Anim Ecol 85:396-408. https://doi. org/10.1111/1365-2656.12441

Papiorek S et al (2015) Bees, birds and yellow flowers: pollinatordependent convergent evolution of UV patterns. Plant Biol $18: 46-55$

Pauw A (2013) Can pollination niches facilitate plant coexistence? Trends Ecol Evol 28:30-37

Potts SG, Vulliamy B, Roberts S, O'Toole C, Dafni A, Ne'eman G, Willmer PG (2004) Nectar resource diversity organises flowervisitor community structure. Entomol Exp Appl 113:103-107

R Core Team (2016) R: a language and environment for statistical computing. R Foundation for Statistical Computing, Vienna

Radville L, Post E, Eissenstat DM (2016) Root phenology in an Arctic shrubgraminoid community: the effects of longterm warming and herbivore exclusion. Clim Change Resp 3:4

Read QD, Moorhead LC, Swenson NG, Bailey JK, Sanders NJ (2014) Convergent effects of elevation on functional leaf traits within and among species. Funct Ecol 28:37-45. https://doi. org/10.1111/1365-2435.12162

Runquist RB, Grossenbacher D, Porter S, Kay K, Smith J (2016) Pollinator-mediated assemblage processes in California wildflowers. J Evol Biol 29:1045-1058. https://doi.org/10.1111/jeb.12845

Sala OE et al (2000) Biodiversity-global biodiversity scenarios for the year 2100. Science 287:1770-1774. https://doi.org/10.1126/ Science.287.5459.1770

Sargent RD, Ackerly DD (2007) Plant-pollinator interactions and the assembly of plant communities. Trends Ecol Evol 23:123-130

Siefert A et al (2015) A global meta-analysis of the relative extent of intraspecific trait variation in plant communities. Ecol Lett 18:1406-1419. https://doi.org/10.1111/ele.12508

Spasojevic MJ, Suding KN (2012) Inferring community assembly mechanisms from functional diversity patterns: the importance 
of multiple assembly processes. J Ecol 100:652-661. https://doi. org/10.1111/J.1365-2745.2011.01945.X

Stang M, Klinkhamer PGL, Meijden E (2007) Asymmetric specialization and extinction risk in plant-flower visitor webs: a matter of morphology or abundance? Oecologia 151:442-453

Stubbs WJ, Wilson JB (2004) Evidence for limiting similarity in a sand dune community. J Ecol 92:557-567. https://doi. org/10.1111/J.0022-0477.2004.00898.X

Sundqvist MK, Sanders NJ, Wardle DA (2013) Community and ecosystem responses to elevational gradients: processes, mechanisms, and insights for global change. Annu Rev Ecol Evol S 44:261280. https://doi.org/10.1146/Annurev-Ecolsys-110512-135750

Swanson HK, Lysy M, Power M, Stasko AD, Johnson JD, Reist JD (2015) A new probabilistic method for quantifying $n$-dimensional ecological niches and niche overlap. Ecology 96:318-324
Swenson NG, Anglada-Cordero P, Barone JA (2011) Deterministic tropical tree community turnover: evidence from patterns of functional beta diversity along an elevational gradient. P R Soc B 278:877-884. https://doi.org/10.1098/Rspb.2010.1369

Tilman D, Knops J, Wedin D, Reich P, Ritchie M, Siemann E (1997) The influence of functional diversity and composition on ecosystem processes. Science 277:1300-1302. https://doi.org/10.1126/ Science.277.5330.1300

Wolowski M, Carvalheiro LG, Freitas L (2017) Influence of plantpollinator interactions on the assembly of plant and hummingbird communities. J Ecol 105:332-344. https://doi. org/10.1111/1365-2745.12684 